\title{
Autoimmunité et interleukines
}

Les interleukines sont des hormones responsables de la communication entre les cellules impliquées dans l'immunité. Des altérations de ces hormones pourraient jouer un rôle important dans l'apparition des maladies autoimmunes.

\section{Annick Harel-Bellan Chargée de recherches Cnrs \\ Didier Fradelizi Directeur de recherches Inserm}

\section{ADRESSE}

A. Harel-Bellan, D. Fradelizi : Laboratoire d'Immunologie, UA 1156 du Cnrs. Institut GusmveRoussy, 94805 Villejuif. es maladies dites autoimmunes regroupent différents types de pathologies dans lesquelles une attaque de l'organisme par son propre système immunitaire peut être mise en évidence. Le lupus érythémateux disséminé (LED), la polyarthrite rhumatoïde (PAR), le syndrome de Sjögren font partie de ce groupe d'affections. Cependant le nombre de maladies dans lesquelles on soupçonne qu'une "autoagression " de l'organisme puisse être à l'origine des symptômes, augmente de jour en jour et s'étend maintenant à certaines formes de diabètes, à la sclérose en plaque et même à certaines maladies parasitaires, telles que la maladie de Chagas ou le paludisme.

L'autoagression peut prendre deux formes. Tantôt des anticorps dirigés contre des "autoantigènes" sont détectés dans le sérum des patients (anémie hémolytique autoimmune, LED, PAR, etc.). Dans d'autres cas, ce sont des lymphocytes T " autoréactifs " qui sont en cause (diabète juvénile, myasthenia gravis, etc.). Ces deux types d'autoagression sont parfois simultanées.

\section{Clones interdits}

L'axe principal d'étude dans ces maladies est la recherche d'un dérèglement du système immunitaire. L'équilibre de ce système est en effet très délicat et vraisemblablement instable. Tout d'abord, au cours du développement de l'embryon, l'organisme apprend à distinguer les molécules du soi des molécules du non-soi. Il est probable que des lymphocytes, capables de reconnaître n'importe quelle structure antigénique, y compris les antigènes du soi, sont génétiquement programmés. Mais les lymphocytes potentiellement autoréactifs doivent être rendus inefficaces. En pratique, ces lymphocytes semblent inactivés mais non éliminés. Ces clones interdits (ainsi sontils dénommés dans le langage familier de l'immunologiste) persistent donc dans l'organisme. On pense que certaines maladies autoimmunes résultent d'une réactivation inappropriée de ces clones interdits à l'occasion d'un dérèglement du système immunitaire encore mal expliqué. C'est en effet une mécanique extrêmement précise et complexe qui maintient l'équilibre du système de telle sorte que l'organisme soit capable de réagir contre les antigènes agresseurs (viraux ou bactériens) sans réagir contre ses propres antigènes. Les clones interdits font l'objet d'une surveillance très stricte par un système de suppression. Le moindre dérèglement de ce système, ou la moindre erreur lors de l'activation de clones au cours d'une réponse normale risque d'aboutir à l'apparition de manifestations autoimmunes.

\section{Régulation}

de la réponse immune

La régulation de la réponse immunitaire n'est pas encore, loin s'en faut, entièrement élucidée. On sait cependant qu'elle correspond à l'interaction entre deux systèmes, un 


\section{REFERENCES}

I. Mizel SB. Interleukin I and T cell activation Immunol Rev 1982; $63: 51-72$.

2. Gillis S, Mochizuki DY, Conlon PJ, et al Molecular caracterization of IL2. Immunol Rev $1982 ; 63: 167-209$.

3. Kishimoto T, Yoshizaki K, Kimoto M, et al $B$ cell growth and differentiation factors and mechanisms of $\mathrm{B}$ cell activation. Immunol Rev $1984 ; 78: 97-118$.

4. Vitetta ES, Brooks $K$, Chen, et al. T cell derived lymphokines that induce IgM and IgG secretion in activated murine B cells. Immunol Rev 1984; $78:$ 137-57

5. Sidman CL, Marshall JD. B cell maturation factor; effect on various cell populations. J Immunol 1984; $132: 845-50$.

6. Leclerq L, Bismuth G, Théze J. Antigen specific helper $T$ cell clone supernatant is sufficien to induce both polyclonal proliferation and differentiation of small resting $B$ lymphocytes. Proc Natl Acad Sci USA 1984; 8I : 649I-5.

7. Chouaib S, Fradelizi D. The mechanism of inhibition of human IL2 production. 7 Immunol 1982; $129: 2463-8$.

8. Andrews BS, Eisenberg RA, Theophilopoulos $A N$, et al. Spontaneous murine lupus-like syndromes; clinical and immunopathological manifestation in several strains. 3 Exp Med 1978; $148: 1198-215$.

9. Moller G. Model of autoimmune diseases Immunol Rev 1981; $55: 1-13$.

10. Scott CF, Tsurufugi M, Lu CY, Finberg $R$ Sy MS. Comparison of antigen-specific $T$ cells responses in autoimmune $\mathrm{MRL} / \mathrm{Mp}-\mathrm{lpr} / \mathrm{lpr}$ and MRL/Mp + / + mice. J Immunol 1984; I32: 633-9.

II. Leventhal B, Talal N. Response of NZB and NZW spleen cells to mitogenic agents. 7 Immunol 1 970; $104:$ 918-23.

12. Ruppert B, Ford R, Maizel A. T cel mediated suppression in the MRL mouse. Cell Immunol 1981; 6I : 40-5.

13. Altman A, Theophilopoulos AN, Weiner $R$, Katz DH, Dixon F. Analysis of T cell function in autoimmune murine strains; defect in production of and responsiveness to IL2. 7 Exp Med 1981 ; $154: 791-808$ système dit hel per, ou auxiliaire, qui déclenche et entretient l'activation des cellules immunocompétentes et un système suppresseur qui, au contraire, freine, voire stoppc ou interdit cette activation. Ces délicats équilibres résultent de l'interaction entre trois principaux types cellulaires : les macrophages, les lymphocytes $\mathrm{T}$ et les lymphocytes B. Parmi ces cellules, les lymphocytes $\mathrm{T}$ semblent jouer le rôle principal de régulation, bien qu'une activité importante puisse également être attribuée aux macrophages. Cette régulation par les lymphocytes $\mathrm{T}$ a lieu dans les deux sens. Il existe en effet, des $\mathrm{T}$ auxiliaires et des T suppresseurs. La communication entre les lymphocytes $T$ régulateurs et les cellules effectrices de l'immunité (lymphocytes $B$ producteurs d'anticorps et lymphocytes $T$ cytolytiques) se fait principalement par le biais de messagers moléculaires. Il s'agit des hormones du système immunitaire, baptisées interleukines pour interrelation entre leukocytes.

Les plus étudiées et les mieux connues de ces molécules sont les hormones dites du help, qui participent au déclenchement et à l'entretien de la réponse. On connaît, en effet, peu de choses sur les facteurs dits suppresseurs qui pourraient participer à l'arrêt de la réponse.

\section{Les interleukines}

Pour expliquer les interactions entre cellules du système immunitaire et l'action des interleukines, nous pouvons décrire brièvement les événements que déclenche l'intrusion dans l'organisme d'un antigène agresseur : bactérie ou virus. Les premières cellules activées par l'antigène sont les macrophages (figure I ). Capables de phagocytose, ils ingèrent l'antigène et ont une double action; d'une part, en le modifiant et en l'exposant à leur membrane, ils présentent l'antigène aux lymphocytes sous la forme d'un complexe antigène-molécule d'histocompatibilité. D'autre part, ils sécrètent une première interleukine, l'interleukine $1\left(\mathrm{IL}_{1}\right)[1]$. C'est alors que les lymphocytes $\mathrm{T}$ sont à leur tour activés (figure 2). En présence du double signal constitué par l'IL 1 et par l'antigène présenté par les macrophages, les lymphocytes $\mathrm{T}$ helper sécrètent une deuxième interleukine, l'interleukine 2 (IL2) [2], qui joue un rôle prépondérant dans le développement de cette seconde phase de la réponse immune. L'IL2 stimule en effet la prolifération et (peut-être) la différenciation des lymphocytes $T$ cytolytiques (CTL) (figure 3 ). Ces CTL sont chargés de la destruction des cellules infectées par un virus. En présence de l'antigène, les précurseurs de ces CTL acquièrent un récepteur pour l'IL2; sous l'action de l'IL2, ils proliferent et se différencient en CTL. Une autre interleukine, l'interféron $\gamma$ (ou immun) agit également au niveau de l'activation des CTL. Ainsi se développe la réponse de type cellulaire. Notons que l'IL2 fait proliférer indistinctement tous les lymphocytes exprimant le récepteur à l'IL2. La réponse immunitaire est cependant très spécifique. En fait, seuls les lymphocytes $T$, spécifiquement capables de reconnaître un antigène donné, sont activés en présence de cet antigène et expriment le récepteur de l'IL2. C'est donc au niveau du mécanisme d'acquisition du récepteur à l'IL2 que se situe le contrôle de la spécificité.

Mais les lymphocytes T helper produisent également de nombreuses autres interleukines. Les plus importantes sont celles présentant un tropisme pour les effecteurs lymphocytaires $B$, responsables de la réponse humorale et donc de la sécrétion d'anticorps. Deux de ces interleukines sont relativement bien décrites à ce jour. La première est le facteur de croissance des lymphocytes B (B Cell Gromth Factor, BCGF) [3] et la seconde, le facteur de différenciation des lymphocytes B (B Cell Differentiation Factor, BCDF) [4]. L'action du BCGF sur les lymphocytes $\mathrm{B}$ est tout à fait à l'image de l'action de l'IL2 sur les lymphocytes $\mathrm{T}$ cytolytiques (voir figure 4). Activés par l'antigène, les lymphocytes $B$ acquièrent un récepteur pour le $\mathrm{BCGF}$ et proliferent en présence de ce facteur. La différenciation de ces cellules en plasmocytes sécréteurs d'anticorps est induite par le BCDF. On connaît peu de choses du mode d'action de cette dernière interleukine, sinon 


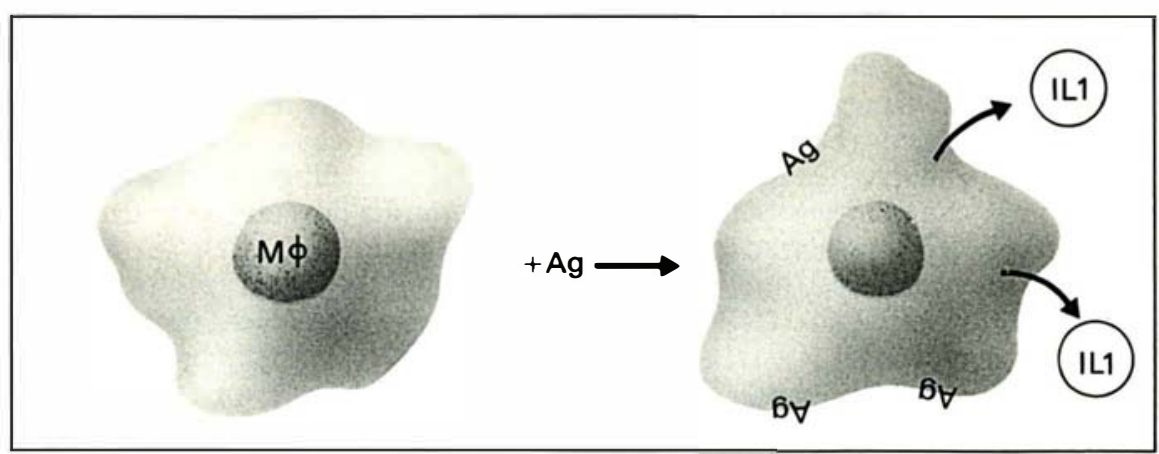

\begin{tabular}{|c|c|}
\hline $\begin{array}{l}\text { MQ } \\
\text { TH } \\
\text { CTL } \\
\text { B } \\
\text { IL1 } \\
\text { IL2 } \\
\text { BCGF } \\
\text { BCDF } \\
\text { INF }\end{array}$ & $\begin{array}{l}\text { :macrophage } \\
\text { :T " helper " } \\
\text { :Iymphocyte T cytolytique } \\
\text { :Iymphocyte B } \\
\text { : interleukine } 1 \\
\text { :interleukine } 2 \\
\text { : B Cell Growth Factor } \\
\text { :B Cell Differentiation } \\
\text { Factor } \\
\text { : interféron }\end{array}$ \\
\hline
\end{tabular}

Figure 1. Activation du macrophage.

Deux effets : le macrophage présente l'antigène à sa surface et il sécrète l'ILI.

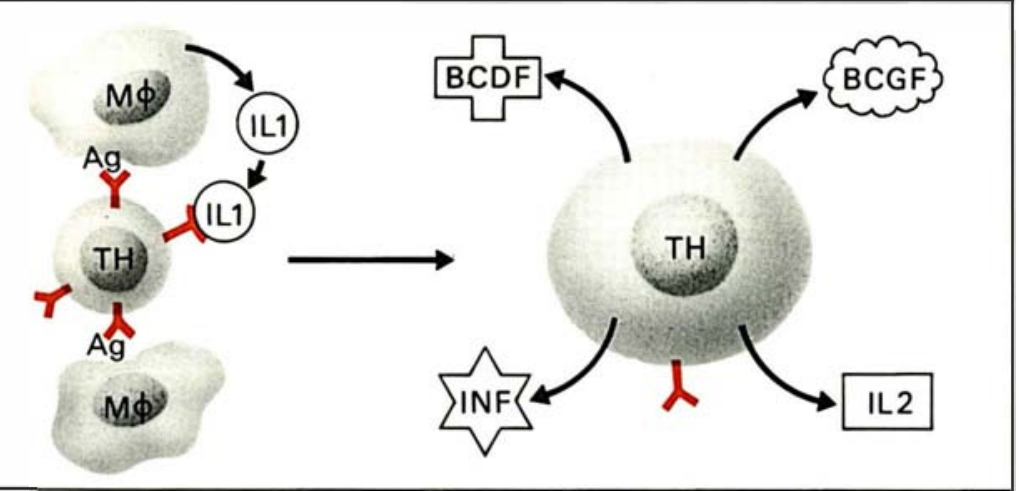

Figure 2. Activation des $T$ " helper".

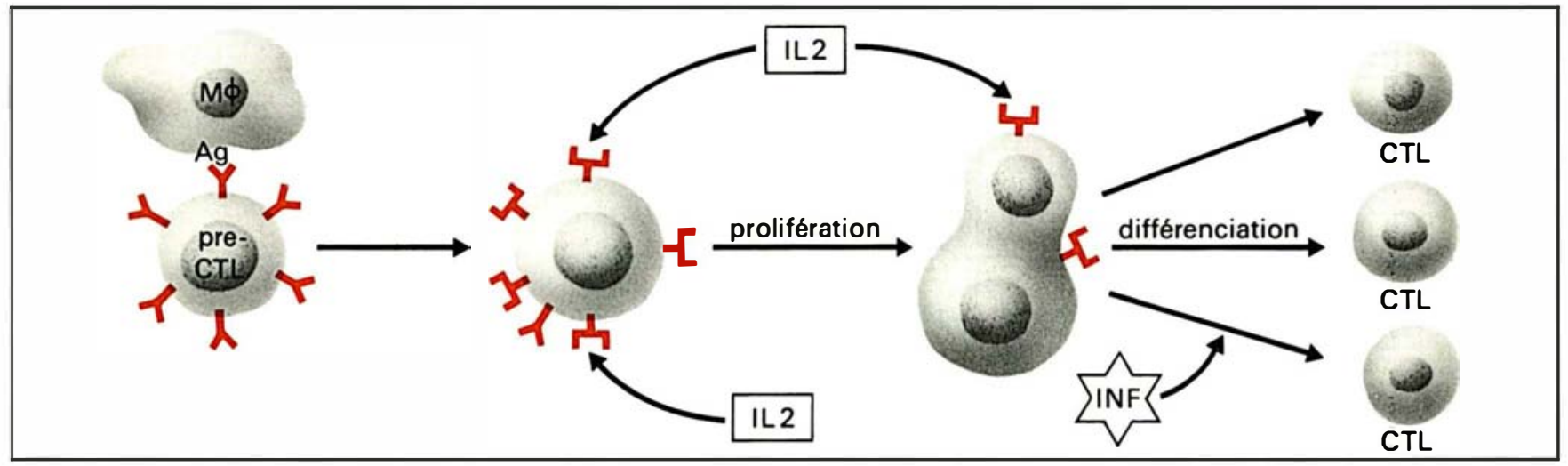

Figure 3. Activation des CTLA

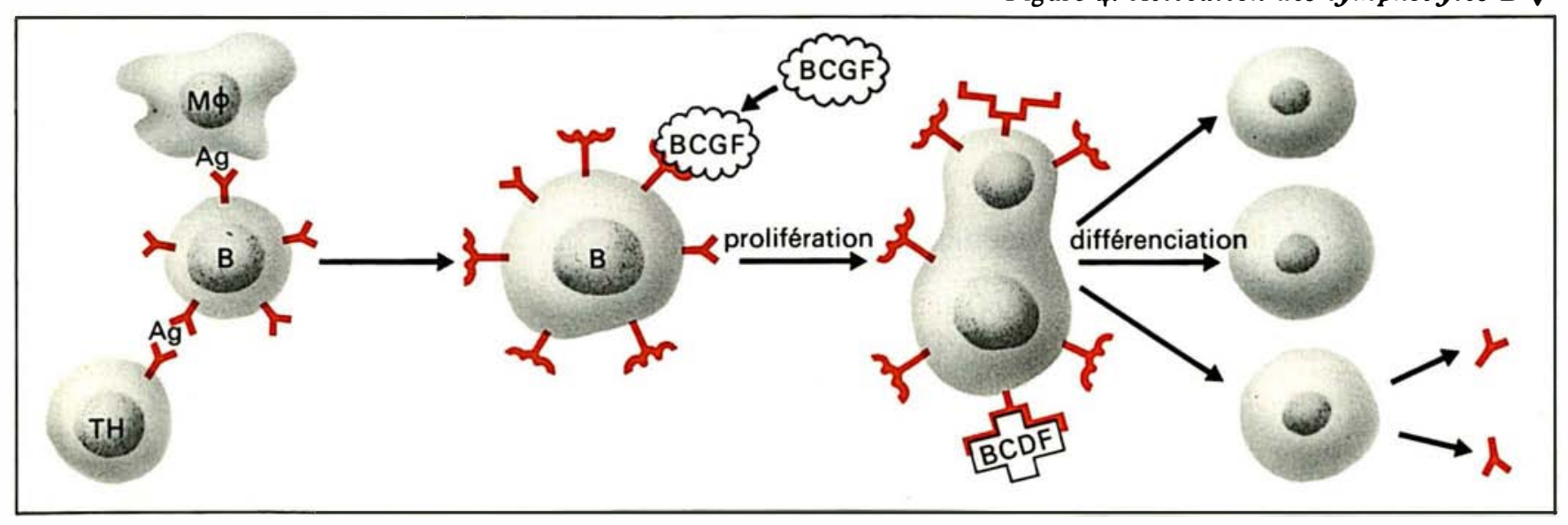




\section{RÉFÉRENCES}

14. Wofsy D, Murphy D, Roths JB, Dauphinee MJ, Kipper B, Talal N. Deficient interleukin 2 activity in $\mathrm{MRL} / \mathrm{Mp}$ and $\mathrm{C}_{57} \mathrm{Br}_{1} / 6$ mice bearing the ipr gene. 7 Exp Med 1981; $154: 1671-80$.

15. Dauphinee MJ, Kipper SB, Wofsy D, Talal $N$. Interleukin 2 deficiency is a common feature of autoimmune mice. 7 Immunol $198 \mathrm{I}$; $127: 2483-7$.

16. Harel-Bellan A, Joskowicz M, Fradelizi D, Eisen $H$. Modification of $T$ cell proliferation and IL2 production in mice infected with Trypanosama cruzi. Proc Natl Acad Sci USA 1983; $80:$ 3466-9.

17. Prudhomme GJ, Fuks A, Colle E, Seemayer TA, Guttmann RD. Immune dysfonction in diabetes-prone BB rats. Interleukin 2 production and other mitogen induced responses are suppressed by activated macrophages. 7 Exp Med 1984; $159: 463-78$.

18. Linker-Israeli M, Bakke AC, Kitridou RC, Gendler S, Gillis S, Horwitz DA. Defective interleukin 1 and interleukin 2 production in patients with systemic lupus erythematosus (SLE). 7 Immunol 1983; $130: 2651-5$.

19. Murakawa Y, Takada S, Ueda Y, Suzuki N, Hoshino T, Sakhane T. Caracterization of T lymphocyte subpopulations responsible for deficient interleukin 2 activity in patients with systemic lupus erythematosus. 7 Immunol 1985; $134: 187-91$.

20. Miyasaka N, Nakamura T, Russell IJ, Talal $N$. Interleukin 2 deficiency in rhumatoid arhtritis and systemic lupus erythematosus. Clin Immunol Immunopathol 1984; B : 109-12.

21. Fradelizi D, Harel-Bellan A, Chatenoud L. Interleukines et autoimmunité. Nouv Presse Méd 1983; $12: 713-6$.

22. Prudhomme GJ, Park CL, Fieser TM, Kofler R, Dixon F J, Theofilopoulos AN. Identification of a B cell differentiation factor(s) spontaneously produced by proliferating cells in murine lupus strains of the lpr/lpr genotype. 7 Exp Med 1983; $157: 730-42$.

23. Sidman CL, Marshall JD, Masiello NC, Roths JB, Shultz LD. Novel B cell maturation factor from spontaneously autoimmune viable motheaten mice. Proc Natl Acad Sci USA 1984; 81 : 7199-202. qu'il existerait plusieurs BCDF, spécifiques des différents types d'immunoglobulines.

Ces facteurs, IL 1 , IL2, BCGF, BCDF ne sont pas les seuls. D'autres interleukines interviennent probablement. Récemment découverte en France et aux Usa, une nouvelle interleukine, active sur les lymphocytes $\mathrm{B}$, dénommée $B$ Cell Activation Factor (BCAF) ou $B$ cell Maturation Factor (BMF) est produite par un certain nombre de clones de lymphocytes $T$ helper cultivés in vitro $[5,6]$. Elle possède la propriété remarquable d'induire la prolifération et la différenciation en plasmocytes sécréteurs d'anticorps de lymphocytes B au repos, n'ayant rencontré aucun antigène. Il s'agit donc d'une activation "polyclonale ", non spécifique. Cette nouvelle interleukine pourrait être importante dans certaines maladies autoimmunes.

Si les cellules et les molécules intervenant dans le déclenchement et l'accélération de la réponse immune commencent à être relativement bien connues, on connaît beaucoup moins bien celles intervenant dans la phase de ralentissement et de suppression de la réponse. Des lymphocytes $\mathrm{T}$ suppresseurs ont été mis en évidence in vitro. Ces lymphocytes proliferent en présence d'IL2 (des clones suppresseurs ont été établis et cultivés en laboratoire). On ne sait cependant pas quel est le rôle exact joué par l'IL2 dans cette phase de ralentissement de la réponse in vivo. Par ailleurs, on a également montré in vitro un rôle suppresseur des macrophages. Cette suppression met en jeu la sécrétion de prostaglandine E2 (PGE2), qui induit l'apparition de $T$ suppresseurs, capables en particulier, de supprimer in vitro la sécrétion d'IL2 par les T helper [7].

On voit que ce système d'interactions lymphocytaires est extrêmement complexe. Encore ceci n'est-il qu'une vue schématique des connaissances actuelles. Ainsi, outre son rôle sur les lymphocytes $T$, l'IL 1 pourrait agir également directement au niveau des lymphocytes B. Par ailleurs, des études très récentes ont montré l'existence de récepteurs pour l'IL2 sur les lymphocytes $B$ activés. Il a également été montré que l'IL2 (hautement purifié ou produit par génie génétique, donc sans équivoque sur la molécule) est capable de faire proliférer des lymphocytes $B$, voire même de les faire sécréter des anticorps. On ne sait pour le moment rien de l'importance physiologique de telles observations faites in vitro. Enfin, très probablement, un grand nombre de facteurs sont encore totalement inconnus.

\section{Interleukines au cours de l'autoimmunité}

Ces interactions complexes commencent à être étudiées dans le cadre des maladies autoimmunes. On espère ainsi déterminer l'étape (ou les étapes) dont le dérèglement pourrait provoquer l'autoimmunité. Les études les plus poussées sur ces interactions ont été réalisées sur des modèles animaux, en particulier chez la souris. L'autoimmunité peut être induite expérimentalement chez l'animal, soit par immunisation dans des conditions particulières avec un autoantigène, soit à la suite de l'infection par certains parasites (Trypanosama Cruzi, Plasmodium...). Les modèles les plus couramment étudiés sont cependant les souris, chez lesquelles le caractère autoimmunité a été sélectionné génétiquement. Chez ces animaux, la maladie apparaît spontanément au cours de la vie. Les souches ainsi sélectionnées (NZB, BXSB, MRL lpr/lpr...) présentent des symptômes divers; elles ont cependant un grand nombre de points communs qui les rapprochent du lupus humain dont elles représentent les modèles animaux [8]. Chez toutes ces souches, on retrouve en effet l'existence d'autoanticorps (en particulier anti-ADN) et l'apparition de glomérulonéphrite.

L'étude de l'état immunitaire de ces souris a donné principalement deux résultats. Tout d'abord, une hyperactivation polyclonale des lymphocytes $B$ a été mise en évidence [9]. Le nombre de lymphocytes $B$ synthétisant activement des anticorps, même en l'absence de toute stimulation antigénique, est en eff et multiplié par un facteur pouvant aller jusqu'à cent chez ces souris. Cette activation polyclonale s'accompagne 
d'une augmentation considérable des Ig circulantes. Deuxième résultat paradoxal, une diminution importante des réponses immunes spécifiques induites par l'immunisation expérimentale. La réponse anticorps contre des immunogènes définis (en particulier les réponses impliquant la coopération des lymphocytes B avec les lymphocytes $T$ ), est très diminuée dans ces souches autoimmunes, tant in vivo qu'in vitro [10].

Parallèlement, les lymphocytes T de ces souris sont incapables de répondre in vitro aux mitogènes (molécules capables d'activer l'ensemble des lymphocytes en mimant une agression antigénique) $[11,12]$. L'étude des interleukines dans ces modèles vient de débuter. Deux observations pouvant être importantes pour la compréhension de ce type de pathologie, ont déjà été rapportées. D'une part, plusieurs équipes ont montré une importante diminution de la production d'IL2 in vitro, par les lymphocytes des souris autoimmunes $[13,14,15]$. Cette observation s'étend à toutes les souches de souris autoimmunes étudiées, ainsi qu'à d'autres modèles, telle la maladie de Chagas expérimentale (infection de souris par un parasite), le Trypanosoma Cruzi (aboutissant également à l'apparition de symptômes autoimmuns) [16], ou le diabète autoimmun du rat [17]. La diminution de la production d'IL2 est quasi totale et précède l'apparition des symptômes cliniques. Cette observation concorde parfaitement avec la suppression des fonctions $T$ observées dans les modèles autoimmuns.

La coïncidence entre la survenue d'une autoimmunité et d'un déficit passager ou durable de l'immunité cellulaire a aussi été notée chez l'homme. Bien que cela reste très hypothétique, ces résultats suggèrent un lien de cause à effet. On pourrait postuler que l'IL2 soit un facteur de croissance indispensable à la prolifération in vivo des lymphocytes $T$ suppresseurs, responsables du blocage des clones interdits, dirigés contre les antigènes du soi. Une diminution de la production d'IL2 entraînerait alors la diminution de ces lymphocytes suppresseurs et l'émergence de ces clones interdits. En revanche, le déficit immunitaire pourrait être secondaire et correspondre à un effet feed-back. Celui-ci résulterait d'une tentative du système pour freiner une hyperactivation pathologique affectant toutes sortes de clones, dont les clones interdits.

Chez l'homme, les études dans les maladies similaires n'ont pas donné des résultats aussi clairs et concordants. Un certain nombre d'équipes prétendaient trouver un effondrement complet de la production d'IL2 in vitro par les lymphocytes circulants de malades atteints de Lupus $[18,19]$. Ces résultats n'ont pas été confirmés par les études récentes qui n'ont montré qu'une faible diminution de la production d'IL2 au cours du Lupus, de la maladie de Sjögren ou de la polyarthrite rhumatoïde $[20,21]$. Il faut cependant noter la grande hétérogénéité des populations étudiées sur le plan des stades de la maladie. Par ailleurs, une étude a mis en évidence des anomalies dans la régulation de la production d'IL2 par les macrophages, via les $\mathrm{PGE}_{2}$. Chez ces malades, les lymphocytes $T$ suppresseurs (de la production d'IL2) ne sont pas activés par les PGE2 sécrétées en grande quantité par leurs monocytes, alors que c'est clairement le cas chez les individus sains [ 21 1].

Une deuxième observation, présentant un grand intérêt pour la compréhension de ce type de pathologie, a également été réalisée chez les souris génétiquement autoimmunes. Les lymphocytes spléniques de ces souris semblent en effet sécréter de façon spontanée et permanente une interleukine $B$-helper, induisant l'activation polyclonale des lymphocytes $B$ au repos [22, 23]. Cette molécule est capable de pousser les lymphocytes à se différencier en plasmocytes sécrétant des anticorps en l'absence de toute activation antigénique. Cette activité vient très récemment d'être décrite dans des situations expérimentales in vitro (dans le surnageant de clones $T$ helper provenant de souris normales; voir cidessus). Cette molécule pourrait bien intervenir au cours des réponses normales, bien que son action aboutisse à la non-spécificité de ces

\section{* GLOSSAIRE*}

Interleukine: Hormone du système immunitaire, permettant la communication entre les lym phocytes el impliquées dans les régulations de la réponse immune.

Interleukine I $\left(I L_{I}\right)$ : Molécule sécrétée par les macrophages lorsqu'ils sont activés. L'ILI est indispensable à lactivation des lymphocytes $T$ " helper .

Interleukine 2 (IL2) : Facteur de croissance des lymphocytes $T$. L'IL2 est sécrétée par les lymphocytes $T$. helper et est indispensable à la croissance des lymphocytes $T$ effecteurs activés.

BCGF (B Cell Growth Factor) : Facieur de croissance des lymphocytes $B$. Le BCGF est sécrété par les lymphocytes $T$ - helper ". Il est indispensable à la croissance des lymphocytes $B$ activés.

BCDF (B Cell Differentiation Factor) : Facteur de différenciation des lymphocytes $B$. Le $B C D F$ est sécrété par les lymphocytes $T$. helper et induit la différenciation des lymphocytes $B$ activés en plasmocytes sécrétant des anticorps. 
réponses. On sait en effet qu'une réponse spécifique s'accompagne fréquemment d'une activation générale du système immunitaire, avec souvent la sécrétion d'immunoglobulines sans fonction anticorps et d'anticorps dont les spécificités correspondent à d'anciennes immunisations. On peut imaginer que, normalement, cette interleukine est sécrétée très tôt dans la réponse, puis rapidement, sa synthèse s'interrompt. Pour une raison encore inconnue, cette synthèse continuerait dans certains cas et aboutirait en permanence à l'activation polyclonale des lymphocytes $\mathrm{B}$ et à l'apparition d'anticorps autoimmuns. Ce modèle est d'autant plus vraisemblable que les maladies autoimmunes surviennent souvent à la suite d'une infection de l'individu. Aucune étude de la production de ce facteur chez l'homme n'a cependant été publiée à ce jour.

\section{Conclusion}

L'implication des interleukines dans la genèse des maladies autoimmunes n'en est qu'à son balbutiement. Elle peut parfois prendre des formes inattendues. Ainsi, outre son rôle dans l'activation de la cytotoxicité cellulaire, l'interféron est également connu pour augmenter la quantité d'antigènes d'histocompatibilité présents à la surface des cellules. On soupçonne qu'il jouerait, par cette action, un rôle dans l'apparition de certaines thyroïdites autoimmunes. Lors d'une infection virale, l'interféron serait sécrété et induirait l'apparition d'antigènes HLA classe II sur les thyrocytes. Ces thyrocytes pourraient alors présenter leurs propres antigènes comme des antigènes étrangers et déclencher ainsi la réponse autoimmune. Il semble donc, qu'étant donné la grande complexité du système (des dérèglements pouvant intervenir à de multiples étapes) et la grande diversité des maladies autoimmunes, aucune découverte ne sera généralisable à tous les cas. Pourtant, parce qu'elle constitue une approche moléculaire, l'étude des interleukines dans ces maladies ouvre des perspectives thérapeutiques prometteuses qui pourraient déboucher sur des applications cliniques intéressantes

\section{Summary}

Autoimmune diseases are those in which the organism attacks himself, with the appearance of autoreactive antibodies and $T$ cells. Dysregulation of the immune system is most probably responsible for this inappropriate response. Interleukins are hormones responsible for the communication between the different cell types involved in immunity. Studies of interleukins in the autoimmune deseases are now in progress. This article contains a schematic view of what is known normally about interleukins and an overview of what is known about these molecules in autoimmunity.

\section{TIRES A PART}

D. Fradelizi, Laboratoire d'Immunologie UA 1156 du Cnrs. Institut Gustave-Roussy, 94805 Villejuif. 